\title{
Dietary Intake and Hypertension Risk: A Cross-Sectional Survey Using Data from the China hypertension survey, 2012-2015
}

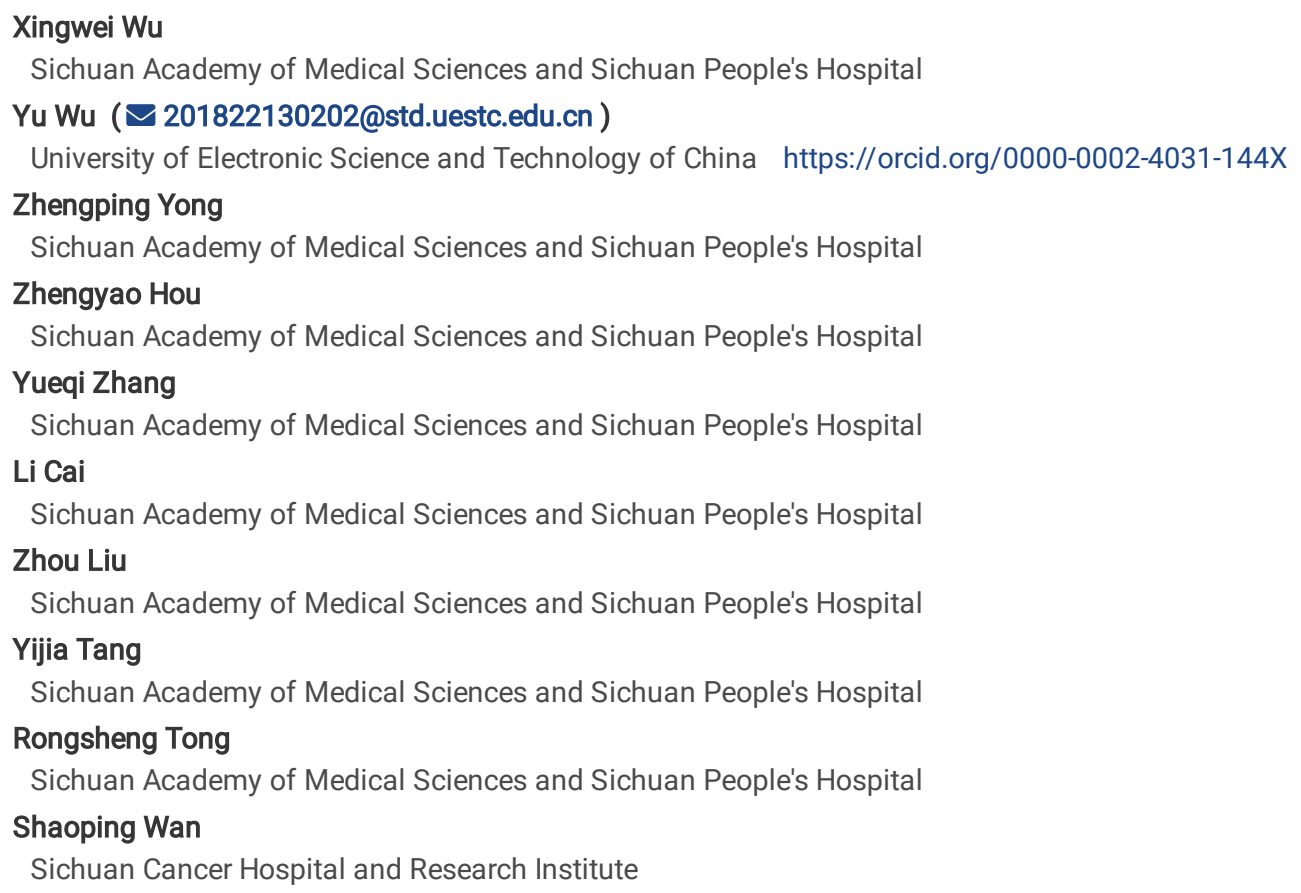




\section{Abstract}

Background: Available data about the effects of dietary intake on hypertension remains limited and conflicting. The purpose of this study was to assess the correlation between dietary intake and the incidence of hypertension.

Methods: The China Hypertension Survey was conducted nationwide, and the data of 19,277 residents from Sichuan was organized, and finally 2,010 samples were selected into this study. Standard food frequency questionnaires were used to collect dietary intake data and physical examinations including blood pressure were performed on site. The subjects were classified into hypertensive group $(n=356)$ and non-hypertensive group ( $n=1,654)$. The odds ratio (OR) for the occurrence of hypertension according to the quartiles of the dietary intake was evaluated by multivariable logistic regression.

Results: After adjustment for all potential confounding variables, more than $50 \mathrm{~g}$ of daily egg intake was a risk factor for hypertension prevalence $(\mathrm{OR}=2.11 ; 95 \% \mathrm{Cl}: 1.34-3.32)$, and $1.94-3.33 \mathrm{~g}$ of animal oil intake per day was a protective factor (OR=0.57; 95\% $\mathrm{Cl}$ : $0.37-0.88)$.

Conclusions: Our findings demonstrate a significant positive correlation between eggs above $50 \mathrm{~g}$ and the risk of hypertension, while $1.94-3.33 \mathrm{~g}$ of animal oil is negatively correlated with the risk of hypertension, indicating that adherence to a small amount of eggs and an appropriate amount of animal oil intake have beneficial effects on the prevention of hypertension.

\section{Introduction}

The prevalence of hypertension (HTN) is high ( 31\% of adults worldwide), which is one of the main causes of cardiovascular disease in adults [1], and also increases the burden of heart attacks, stroke, kidney failure, disability and even premature mortality. Researchers have estimated that raised blood pressure generally causes nine million deaths every year[2], partly due to inadequacies in prevention, diagnosis, and control [3]. Early interventions make it possible to minimize the risk of heart attack, stroke and other diseases. For patients, it was reported to be cheaper and safer than interventions like cardiac bypass operation or dialysis that may be needed when HTN is not prevented or treated in time[4]. Therefore, the identification of risk factors related to HTN is of great significance for the prevention and control of HTN. Studies have shown that many risk factors are associated with HTN[5], some risk factors cannot be prevented such as family history and age[6], unhealthy diet is another risk factor, and it is completely preventable[7]. Diet is closely related to energy intake, and which is related to weight control and the maintenance of organisms[8]. Thus, identifying preventable dietary factors is necessary to prevent and control HTN.

Some researchers found eating abundant amounts of fish, vegetables, and low-fat dairy products, and limited intake of sugar foods, red meat and added fats could help prevent HTN[9]. In fact, in the past two decades, health experts have recommended a dietary approach to achieve prevention and control of cardiovascular diseases by increasing the consumption of fruits, cereals, vegetables and low-fat dairy products, and reducing the consumption of fat, red meat, sodium and sugar [10-12]. However, the prevalence of HTN has continued to increase over the years[13], and a large number of researchers have assessed the effect of different dietary intake on the risk of HTN [8, 14], but no final conclusion could be drawn.

Therefore, this study was carried out to explore the correlation between dietary intake and HTN using data from the 2012-2015 China Hypertension Survey and provide a diet-related basis for the prevention of HTN.

\section{Methods Study Design}

From October 2012 to December 2015, Chinese Hypertension Survey was conducted across the country, which aimed to clarify the status of major cardiovascular diseases and major risk factors in the Chinese population, and to provide a scientific basis for the development of cardiovascular disease prevention strategies, the data of Sichuan Province was used in this study. Data of demographic characteristics and dietary intake of the participants were obtained using questionnaires, while blood pressure were obtained by physical examinations. The study has passed the review and approval of the Ethics Committee of Fuwai Hospital (Beijing, China) and obtained the informed consent signed by each participant. Details concerning study protocol have been reported elsewhere [15].

Stratified multistage random sampling method was used for the survey. First, the 31 provinces in China were regarded as the main stratum, and in each province, they were divided into 2 layers: urban and rural. According to administrative data, all counties in the country were defined as rural areas, and all districts were defined as cities. Participants in each layer were selected using a four-stage random sampling method. The first stage: 4 cities/counties were selected by the probability proportional to size (PPS) method in each of the 62 layers. The second stage: the simple random sampling (SRS) method was used to select 2 districts/townships in each city/county. The third stage: the SRS method was also used to select 3 communities/villages in each district/township. The fourth stage: in each selected community/village, the SRS method was used to select a given number of individuals according to gender (male and female) and age groups (15-24, 25-34, 35-44, 45-54, 55-64, 65-74 and $\geq 75$ ).

In order to explore the factors related to the prevalence of HTN, essential data on gender (male or female), age (55 years old or $\geq 55$ years old), education (primary school and below or above), marriage (single or married), employment status (employed or unemployed), agricultural workers (yes or no), family history of HTN (yes or no), elderly over 80 years old in the family (with or without), weight (kg), height (cm), alcohol consumption (yes or 
no), smoking status (non-smokers, past smokers or current smokers), pulse (bpm) and blood pressure were collected. The family history of HTN was determined by whether the participant's grandparents, parents, siblings, or children suffering from HTN. Employment status was classified into employed and unemployed. Unemployment included retirees, students and idle personnel.

\section{Definition of htn}

According to the 2018 Chinese prevention and treatment guidelines of HTN [16], it is defined as HTN if one of the following two criteria was met. (1) using antihypertensive medications within 2 weeks, (2) systolic blood pressure (SBP) $\geq 140 \mathrm{~mm} \mathrm{Hg}$ or diastolic blood pressure (DBP) $\geq 90 \mathrm{~mm} \mathrm{Hg}$.

In the study, participants who suffered from HTN were defined as having a self-reported diagnosis of HTN or having used anti-hypertensive medication within 2 weeks or the average blood pressure was higher than 140/90 $\mathrm{mmHg}$ measured during the physical examination.

\section{Anthropometrical And Blood Pressure Measurements}

The pulse and blood pressure of each participant's right arm were measured three times with 30-second intervals using a OMRON HBP-1300 Professional Portable Blood Pressure Monitor (OMRON, Kyoto, Japan) after the participant sat still for five minutes. The average values were calculated for statistical analysis. The previous research has confirmed that Omron HBP-1300 used for BP measurement was accurate [17].

Height was measured using a stadiometer in a standing position without shoes and hats, keeping shoulders flat and arms drooping naturally. Weight was measured using an electronic scale while the participants wore the least clothes and stood barefoot on the instrument. Combined with the participant's weight and height, the BMI (Body Mass Index, $\mathrm{kg} / \mathrm{m}^{2}$ ) value could be easily obtained.

\section{Dietary Intake Assessment}

To assess whether diet is related to HTN, a standardized questionnaire was designed by the Fuwai Hospital to obtain data on the intake quantity and frequency of various foods by participants in the past year, including cereals, potatoes, vegetables, livestock meat, poultry meat, fish, aquatic products, eggs, milk, bean products, fruits, nuts, pickles, vegetable oil, animal oil and salt. To make it easier for participants to answer, intake quantity was described by the units commonly used in China: "jin" ( 1 jin equals 500 grams) and "liang" ( 1 liang equals 50 grams). The intake frequency was categorized into five categories: never, daily, weekly, monthly and yearly. The average daily food intake was calculated as the total intake quantity of each food divided by the days corresponding to the frequency of food intake. The questionnaire only recorded the monthly intake of vegetable oil, animal oil and salt for a family. The daily intake was equal to vegetable oil, animal oil and salt monthly intake quantity divided by 30 days and then divided by the number of people dining at home.

\section{Statistical analysis}

Data of food intake was analyzed in continuous and categorical form, and the latter was classified according to the quartile of food intake. Among them, the values at the $25 \%$ and $75 \%$ positions of the variable livestock meat are the same. Each kind of food included all foods in that category, for example, potatoes including sweet potatoes, yams and taros, vegetables included all kinds of vegetables except potatoes, eggs came from various oviparous animals such as chicken, duck, goose and quail, Nuts included plant seeds such as peanuts and walnuts and other hard nuts.

Continuous variables were presented as the mean \pm SD (standard deviation), and categorical variables were presented as frequencies and percentages. Comparative analyses of continuous variables were analyzed using variance analysis or rank-sum test as appropriate. Comparisons of the categorical variables were analyzed by Chi-square or Fisher's exact test as appropriate. Continuous variables were converted to categorical variables according to their quartiles and entered into the models. Three logistic regression models were built for risk factors analysis. Likelihood ratio test and Akaike's Information Criteria (AIC) were used to evaluate global null hypothesis and goodness-of-fit of the three models respectively. Model 1 was crude and only included different kinds of food. Model 2 was adjusted for age and gender. Model 3 was further adjusted for education background, marital, employment, occupational, alcohol consumption and smoking status, family history of HTN, family members $\geq 80$ years old who are still alive, BMI and pulse. The odds ratios (ORs) before and after adjustment and their $95 \%$ confidence intervals ( $95 \%$ Cls) for the parameters were estimated. A two-tailed $P$ value was used in the study, $\mathrm{P}<0.05$ was considered statistically significant. All statistical analyses were conducted using SAS version 9.4 software (SAS institute INC, Cary, NC, USA)

\section{Results}

\section{Study Sample}

A total of 19,277 valid questionnaires were obtained for subjects aged 15 and above. Participants who did not record complete demographic characteristics and dietary intake history were excluded, finally, a total of 2,010 participants were selected and classified into the hypertensive group (n = 356) and the non-hypertensive group $(n=1,654)$ according to the definition of HTN (Fig. 1). 


\section{Demographic And Dietary Characteristics Of The Study Population}

In this study, the data from these 2,010 participants were used for statistical analysis. Among these people, 641 (31.89\%) were $\geq 55$ years old and 1,012 (50.35\%) were males. The mean (standard deviation, SD) SBP and DBP of all subjects were $121.53(17.32) \mathrm{mmHg}$ and $72.17(11.15) \mathrm{mmHg}$, respectively (Table 1$)$.

Table 1

Characteristics of study participants

\begin{tabular}{|c|c|c|c|c|}
\hline Characteristics & Total Population $(n=2010)$ & Without HTN $(n=1654)$ & With HTN $(n=356)$ & P Value \\
\hline Male & 1012(50.35) & $829(50.12)$ & 183(51.40) & 0.660 \\
\hline Age $\geq 55$ years & $641(31.89)$ & $369(22.31)$ & $272(76.40)$ & $<0.0001^{*}$ \\
\hline Above elementary school & $1265(62.94)$ & 1163(70.31) & $102(28.65)$ & $<0.0001^{\star}$ \\
\hline Married & 1557(77.46) & $1277(77.21)$ & $280(78.65)$ & 0.554 \\
\hline Employed & $1115(55.47)$ & $965(58.34)$ & $150(42.13)$ & $<0.0001^{\star}$ \\
\hline Agricultural workers & $1018(50.65)$ & $722(43.65)$ & 296(83.15) & $<0.0001^{\star}$ \\
\hline Family history of HTN & $312(15.52)$ & $230(13.91)$ & $82(23.03)$ & $<0.0001^{\star}$ \\
\hline Elderly people over 80 in the family & $316(15.72)$ & $261(15.78)$ & $55(15.45)$ & 0.877 \\
\hline BMI(Body Mass Index, kg/m²) & & & & $<0.0001^{\star}$ \\
\hline$<18.5$ & 203(10.10) & 187(11.31) & 16(4.49) & \\
\hline $18.5-23.9$ & 1077(53.58) & $928(56.11)$ & 149(41.85) & \\
\hline $24.0-27.9$ & $568(28.26)$ & $433(26.18)$ & 135(37.92) & \\
\hline$\geq 28.0$ & $162(8.06)$ & $106(6.41)$ & $56(15.73)$ & \\
\hline Alcohol consumption & $485(24.13)$ & $397(24.00)$ & $88(24.72)$ & 0.774 \\
\hline Smoking status & & & & 0.0746 \\
\hline Non-smokers & $1390(69.15)$ & $1160(70.13)$ & $230(64.61)$ & \\
\hline Past smokers & $38(1.89)$ & $28(1.69)$ & $10(2.81)$ & \\
\hline Current smokers & $582(28.96)$ & $466(28.17)$ & 116(32.58) & \\
\hline Pulse, bpm & $77.19 \pm 9.63$ & $77.17 \pm 9.37$ & $77.25 \pm 10.78$ & 0.840 \\
\hline $\mathrm{SBP}, \mathrm{mmHg}$ & $121.53 \pm 17.32$ & $116.23 \pm 11.17$ & $146.17 \pm 19.39$ & $<0.0001^{*}$ \\
\hline DBP, mmHg & $72.17 \pm 11.15$ & $70.40 \pm 9.67$ & $80.40 \pm 13.62$ & $<0.0001^{\star}$ \\
\hline
\end{tabular}

Table S1 reports the baseline characteristics of participants in this study for different intake categories of total whole cereals, potatoes, vegetables, livestock meat, poultry meat, fish, aquatic products, eggs, milk, bean products, fruits, nuts, pickles, vegetable oil, animal oil and salt. The number of men and family history of HTN increased across cereal consumption quartiles. Education level and the number of non-agricultural workers increased across potato consumption quartiles, whereas alcohol consumption decreased. As the quartile of vegetable consumption increased, more participants were married and agricultural workers; more participants were male, non-agricultural workers, and did not drink alcohol with the quartile of livestock meat consumption increased; more participants were male, $<55$ years old, above elementary school education, and employed with the quartile of livestock meat consumption increased. People with higher intake of fish were $<55$ years old and above elementary school; people with higher intake of aquatic product were $<55$ years old, above elementary school, employed and non-agricultural workers; people with higher intake of fruit were female, $<55$ years old, above elementary school, non-agricultural workers, and did not smoke. The population with higher fruit intake was female, $<55$ years old, above elementary school, non-agricultural workers, and did not smoke; the group with higher milk intake was female, above elementary school, nonagricultural workers, and did not drink alcohol. Participants without drinking alcohol had higher egg intake. Non-agricultural workers ate more bean products. The population with higher nut intake was male and non-agricultural workers, and had higher diastolic blood pressure. Participants with higher intake of animal oil were employed.

The participants' diet was mainly cereals and vegetables, followed by livestock meat and fruits (Table 2). 
Table 2

Dietary intake of study participants in the past year (in continuous and categorical form), $\mathrm{g} / \mathrm{day}$

\begin{tabular}{|c|c|c|c|c|}
\hline Dietary intake & Total Population $(n=2010)$ & Without HTN $(n=1654)$ & With HTN $(n=356)$ & P Value \\
\hline Cereal & $217.10 \pm 80.54$ & $216.26 \pm 79.25$ & $220.98 \pm 86.33$ & 0.505 \\
\hline$\leq 150.00$ & 645(32.09) & $523(31.62)$ & $122(34.27)$ & \\
\hline $150.00-200.00$ & $471(23.43)$ & $404(24.43)$ & $67(18.82)$ & \\
\hline $200.00-250.00$ & 498(24.78) & $413(24.97)$ & $85(23.88)$ & \\
\hline$>250.00$ & 396(19.70) & $314(18.98)$ & 82(23.03) & 0.0673 \\
\hline Potato & $23.35 \pm 21.84$ & $23.59 \pm 22.06$ & $22.25 \pm 20.76$ & 0.307 \\
\hline$\leq 6.67$ & $549(27.31)$ & $447(27.03)$ & 102(28.65) & \\
\hline $6.67-16.67$ & $566(28.16)$ & $468(28.3)$ & $98(27.53)$ & \\
\hline $16.67-35.71$ & $377(18.76)$ & $315(19.04)$ & $62(17.42)$ & \\
\hline$>35.71$ & $518(25.77)$ & $424(25.63)$ & $94(26.4)$ & 0.8431 \\
\hline Vegetable & $261.06 \pm 113.01$ & $259.95 \pm 113.41$ & $266.17 \pm 111.17$ & 0.271 \\
\hline$\leq 200.00$ & 732(36.42) & 613(37.06) & 119(33.43) & \\
\hline $200.00-250.00$ & 688(34.23) & $559(33.8)$ & $129(36.24)$ & \\
\hline $250.00-300.00$ & $91(4.53)$ & $75(4.53)$ & $16(4.49)$ & \\
\hline$>300.00$ & $499(24.83)$ & $407(24.61)$ & $92(25.84)$ & 0.6252 \\
\hline Livestock meat & $120.63 \pm 56.14$ & $122.43 \pm 56.23$ & $112.29 \pm 55.03$ & $0.002^{\star}$ \\
\hline$\leq 100.00$ & 1192(59.30) & $962(58.16)$ & $230(64.61)$ & \\
\hline $100.00-150.00$ & $458(22.79)$ & $381(23.04)$ & $77(21.63)$ & \\
\hline$>150.00$ & $360(17.91)$ & $311(18.8)$ & $49(13.76)$ & $0.0396^{*}$ \\
\hline Poultry meat & $24.97 \pm 23.59$ & $25.65 \pm 23.70$ & $21.82 \pm 22.83$ & $0.001^{\star}$ \\
\hline$\leq 6.67$ & $543(27.01)$ & $430(26)$ & 113(31.74) & \\
\hline $6.67-16.67$ & $556(27.66)$ & $447(27.03)$ & 109(30.62) & \\
\hline $16.67-35.71$ & $403(20.05)$ & $346(20.92)$ & $57(16.01)$ & \\
\hline$>35.71$ & $508(25.27)$ & $431(26.06)$ & $77(21.63)$ & $0.0132 *$ \\
\hline Fish & $17.21 \pm 15.86$ & $18.25 \pm 16.01$ & $12.37 \pm 14.18$ & $<0.0001^{*}$ \\
\hline$\leq 5.00$ & $541(26.92)$ & $387(23.4)$ & 154(43.26) & \\
\hline $5.00-14.29$ & $465(23.13)$ & $395(23.88)$ & $70(19.66)$ & \\
\hline $14.29-25.00$ & $547(27.21)$ & $461(27.87)$ & $86(24.16)$ & \\
\hline$>25.00$ & 457(22.74) & $411(24.85)$ & $46(12.92)$ & $<0.0001^{*}$ \\
\hline Aquatic product & $1.74 \pm 2.25$ & $1.86 \pm 2.30$ & $1.17 \pm 1.92$ & $<0.0001^{*}$ \\
\hline$\leq 0.00$ & $510(25.37)$ & $370(22.37)$ & $140(39.33)$ & \\
\hline $0.00-0.68$ & 493(24.53) & $408(24.67)$ & $85(23.88)$ & \\
\hline $0.68-2.74$ & $512(25.47)$ & $440(26.6)$ & $72(20.22)$ & \\
\hline$>2.74$ & $495(24.63)$ & $436(26.36)$ & $59(16.57)$ & $<0.0001 *$ \\
\hline Egg & $30.24 \pm 32.21$ & $29.16 \pm 31.01$ & $35.26 \pm 36.91$ & 0.184 \\
\hline$\leq 6.67$ & $504(25.07)$ & $408(24.67)$ & $96(26.97)$ & \\
\hline $6.67-16.67$ & $609(30.30)$ & $513(31.02)$ & $96(26.97)$ & \\
\hline $16.67-50.00$ & $542(26.97)$ & $465(28.11)$ & $77(21.63)$ & \\
\hline
\end{tabular}

$* \mathrm{P}<0.05$. Data are presented as mean $\pm \mathrm{SD}$ for continuous variables and $\%$ for categorical variables. 


\begin{tabular}{|c|c|c|c|c|}
\hline Dietary intake & Total Population $(n=2010)$ & Without HTN $(n=1654)$ & With HTN $(n=356)$ & P Value \\
\hline$>50.00$ & $355(17.66)$ & $268(16.2)$ & $87(24.44)$ & $0.0004^{*}$ \\
\hline Milk & $82.93 \pm 95.51$ & $84.18 \pm 94.42$ & $77.11 \pm 100.35$ & $0.001 *$ \\
\hline$\leq 6.85$ & $507(25.22)$ & $378(22.85)$ & $129(36.24)$ & \\
\hline $6.85-35.71$ & $417(20.75)$ & $361(21.83)$ & $56(15.73)$ & \\
\hline $35.71-142.86$ & 616(30.65) & $524(31.68)$ & $92(25.84)$ & \\
\hline$>142.86$ & $470(23.38)$ & $391(23.64)$ & $79(22.19)$ & $<0.0001^{*}$ \\
\hline Bean product & $12.60 \pm 12.08$ & $12.66 \pm 12.03$ & $12.33 \pm 12.33$ & 0.325 \\
\hline$\leq 3.33$ & $325(16.17)$ & $253(15.3)$ & $72(20.22)$ & \\
\hline $3.33-7.14$ & 637(31.69) & $534(32.29)$ & $103(28.93)$ & \\
\hline $7.14-16.67$ & $552(27.46)$ & $457(27.63)$ & $95(26.69)$ & \\
\hline$>16.67$ & $496(24.68)$ & $410(24.79)$ & $86(24.16)$ & 0.1338 \\
\hline Fruit & $132.89 \pm 125.98$ & $140.80 \pm 126.38$ & $96.17 \pm 117.48$ & $<0.0001^{*}$ \\
\hline$\leq 33.33$ & $499(24.83)$ & $353(21.34)$ & $146(41.01)$ & \\
\hline $33.33-100.00$ & $617(30.70)$ & $517(31.26)$ & $100(28.09)$ & \\
\hline $100.00-200.00$ & $404(20.10)$ & $355(21.46)$ & $49(13.76)$ & \\
\hline$>200.00$ & $490(24.38)$ & $429(25.94)$ & 61(17.13) & $<0.0001^{*}$ \\
\hline Nut & $9.66 \pm 10.32$ & $9.75 \pm 10.15$ & $9.28 \pm 11.07$ & $0.001 *$ \\
\hline$\leq 1.67$ & $520(25.87)$ & $391(23.64)$ & $129(36.24)$ & \\
\hline $1.67-5.00$ & $491(24.43)$ & $422(25.51)$ & 69(19.38) & \\
\hline $5.00-14.29$ & $589(29.30)$ & $509(30.77)$ & $80(22.47)$ & \\
\hline$>14.29$ & $410(20.40)$ & $332(20.07)$ & 78(21.91) & $<0.0001 *$ \\
\hline Pickle & $12.14 \pm 12.69$ & $12.20 \pm 12.54$ & $11.83 \pm 13.40$ & $0.047 *$ \\
\hline$\leq 1.67$ & $554(27.56)$ & $437(26.42)$ & 117(32.87) & \\
\hline $1.67-7.14$ & $375(18.66)$ & $314(18.98)$ & 61(17.13) & \\
\hline $7.14-16.67$ & $710(35.32)$ & 601(36.34) & 109(30.62) & \\
\hline$>16.67$ & $371(18.46)$ & $302(18.26)$ & 69(19.38) & $0.0489 *$ \\
\hline Vegetable oil & $41.19 \pm 12.76$ & $41.31 \pm 12.59$ & $40.64 \pm 13.52$ & 0.351 \\
\hline$\leq 33.33$ & $357(17.76)$ & $288(17.41)$ & 69(19.38) & \\
\hline $33.33-41.49$ & $474(23.58)$ & $387(23.4)$ & $87(24.44)$ & \\
\hline $41.49-50.00$ & $843(41.94)$ & 702(42.44) & 141(39.61) & \\
\hline$>50.00$ & $336(16.72)$ & 277(16.75) & $59(16.57)$ & 0.7193 \\
\hline Animal oil & $2.07 \pm 2.29$ & $2.04 \pm 2.18$ & $2.21 \pm 2.71$ & 0.369 \\
\hline$\leq 0.00$ & $724(36.02)$ & $568(34.34)$ & $156(43.82)$ & \\
\hline $0.00-1.94$ & 253(12.59) & 217(13.12) & $36(10.11)$ & \\
\hline $1.94-3.33$ & $492(24.48)$ & $436(26.36)$ & $56(15.73)$ & \\
\hline$>3.33$ & $541(26.92)$ & $433(26.18)$ & 108(30.34) & $<0.0001^{*}$ \\
\hline Salt & $4.78 \pm 1.78$ & $4.72 \pm 1.74$ & $5.02 \pm 1.97$ & $0.024^{*}$ \\
\hline$\leq 3.33$ & $323(16.07)$ & 261(15.78) & $62(17.42)$ & \\
\hline $3.33-4.78$ & 992(49.35) & $843(50.97)$ & 149(41.85) & \\
\hline $4.78-5.56$ & $244(12.14)$ & $209(12.64)$ & $35(9.83)$ & \\
\hline
\end{tabular}




\begin{tabular}{|lllll|}
\hline Dietary intake & Total Population $(\mathbf{n = 2 0 1 0 )}$ & Without HTN $(\mathbf{n = 1 6 5 4 )}$ & With HTN $(\mathbf{n = 3 5 6 )}$ & P Value \\
\hline$>5.56$ & $451(22.44)$ & $341(20.62)$ & $110(30.9)$ & $<0.0001$ * \\
\hline *P $<0.05$. Data are presented as mean \pm SD for continuous variables and \% for categorical variables. \\
\hline
\end{tabular}

\section{Differences in demographic and clinical characteristics between HTN and non-HTN groups}

Compared with 1,654 non-hypertensive subjects, hypertensive subjects ( $n=356,17.71 \%$ ) were more likely to be 50 years old and above, unemployed, engaged in agriculture (all $\mathrm{P}<0.0001$ ), and they were more likely to have a lower education, have a family history of HTN, and have a higher body mass index (BMI), the differences were statistically significant (all $\mathrm{P}<0.0001)$. No significant difference was observed in gender, marital status, drinking, smoking, pulse and whether there were elderly people over 80 years old in the family (all $P>0.05)($ Table 1$)$.

\section{Differences in dietary intake between HTN group and non-HTN group}

When the daily food intake in the past year in Table 2 was expressed as continuous, compared with non-hypertensive group, participants with HTN consumed less livestock meat $(P=0.002)$, poultry meat $(P=0.01)$, fish $(P<0.0001)$, aquatic products $(P<0.0001)$, milk $(P=0.001)$, fruit $(P<0.0001)$, nut $(P=0.001)$ but more pickle $(P=0.047)$ and salt $(P=0.024)$. When we converted the continuous variable according to the quartile of food intake into a categorical variable, The differences in the intake of livestock meat $(P=0.0396)$, poultry meat $(P=0.0132)$ and pickle $(P=0.0489)$ between the two groups of participants were less significant while the differences in milk $(P<0.0001)$, nut $(P<0.0001)$ and salt $(P<0.0001)$ were more significant.

In addition, the difference in egg and animal oil intake between participants with and without HTN became significant $(P=0.0004$ and $<0.0001$, respectively), which might be caused by the bias of data distribution. Among the lowest and highest quartiles of egg daily intake, the hypertensive group had a higher percentage than the non-hypertensive group (the lowest quartile: with HTN $26.97 \%$, without HTN $24.67 \%$; the highest quartile: with HTN 24.44\%, without HTN 16.2\%), while in the second and third quartiles, the hypertensive group had a lower percentage of people (the second quartile: with HTN 26.97\%, without HTN 31.02\%; the third quartile: with HTN 21.63\%, without HTN 28.11\%). And among the lowest and highest quartiles of animal oil daily intake, the hypertensive group also had a higher percentage of people than the non-hypertensive group (the lowest quartile: with HTN 43.82\%, without HTN 34.34\%; the highest quartile: with HTN 30.34\%, without HTN $26.18 \%$ ), while in the second and third quartiles, the hypertensive group had a lower percentage of people (the second quartile: with HTN $10.11 \%$, without HTN $13.12 \%$; the third quartile: with HTN $15.73 \%$, without HTN $26.36 \%)$.

\section{Multivariate Analysis of Correlation between HTN and Dietary Intake after Variable Adjustment}

The results of logistic regression analysis with the endpoint of HTN in three distinct models are shown in Table 3 . With the increase of adjusted factors, the AIC values of the three models decreased successively, which were 1747.174, 1535.316 and 1429.012, respectively, showed that the model 3 with the smallest AIC was considered satisfactory. And the covariables considered by the models were meaningful (in testing the Global Null Hypothesis, all values are $\mathrm{P}<0.0001$ ). Before adjustment, the intake of fish, aquatic product, egg, milk, fruit, nut and animal oil had significant correlations with $\mathrm{HTN}$. Among them, the daily intake of egg $>50 \mathrm{~g}$ was a risk factor for $\mathrm{HTN}$, and other variables were protective factors. In model 1 , daily intake $>50 \mathrm{~g}$ of egg was still a risk factor for $\mathrm{HTN}(\mathrm{OR}=1.82,95 \% \mathrm{Cl}=1.18-2.80)$, milk and animal oil intake were still protective factors that were significantly related to HTN, while other variables became insignificant. In model 2, compared with people who daily consume $\leq 6.67 \mathrm{~g}$ egg, people who consume $>50 \mathrm{~g}$ daily might be more likely to suffer from $\mathrm{HTN}(\mathrm{OR}=2.11,95 \square \mathrm{Cl}=1.34-3.32)$. Compared with daily intake of $0 \mathrm{~g}$ of animal oil, $1.94-3.33 \mathrm{~g}$ might be more secure $(O R=0.57,95 \square \mathrm{Cl}=0.37-0.88)$, this group of people might be less susceptible to HTN. And all of these correlations existed independently. 
Table 3

Association between dietary intake and hypertension prevalence, $\mathrm{g} /$ day

\begin{tabular}{|c|c|c|c|c|c|c|}
\hline Dietary intake & Model 1 & & Model 2 & & Model 3 & \\
\hline & $\mathrm{OR}(95 \% \mathrm{Cl})$ & $\mathrm{P}$ & $\mathrm{OR}(95 \% \mathrm{Cl})$ & $\mathrm{P}$ & $\mathrm{OR}(95 \% \mathrm{Cl})$ & $\mathrm{P}$ \\
\hline Cereal & & 0.210 & & 0.527 & & 0.489 \\
\hline$\leq 150.00$ & 1.00 (reference) & & 1.00 (reference) & & 1.00 (reference) & \\
\hline $150.00-200.00$ & $0.88(0.62-1.26)$ & & $0.94(0.64-1.38)$ & & $0.82(0.54-1.24)$ & \\
\hline $200.00-250.00$ & $1.02(0.72-1.45)$ & & $1.02(0.70-1.50)$ & & $0.85(0.56-1.28)$ & \\
\hline$>250.00$ & $1.34(0.93-1.94)$ & & $1.28(0.86-1.92)$ & & $1.12(0.73-1.72)$ & \\
\hline Potato & & 0.120 & & 0.286 & & 0.239 \\
\hline$\leq 6.67$ & 1.00 (reference) & & 1.00 (reference) & & 1.00 (reference) & \\
\hline $6.67-16.67$ & $1.20(0.83-1.73)$ & & $1.19(0.80-1.77)$ & & $1.16(0.77-1.75)$ & \\
\hline $16.67-35.71$ & $1.31(0.87-1.97)$ & & $1.43(0.92-2.22)$ & & $1.43(0.89-2.27)$ & \\
\hline$>35.71$ & $1.65(1.09-2.51)^{\star}$ & & $1.48(0.94-2.33)$ & & $1.57(0.97-2.53)$ & \\
\hline Vegetable & & 0.316 & & 0.852 & & 0.777 \\
\hline$\leq 200.00$ & 1.00 (reference) & & 1.00 (reference) & & 1.00 (reference) & \\
\hline $200.00-250.00$ & $1.27(0.94-1.73)$ & & $1.15(0.83-1.60)$ & & $1.06(0.75-1.49)$ & \\
\hline $250.00-300.00$ & $1.48(0.76-2.86)$ & & $1.15(0.56-2.35)$ & & $0.90(0.42-1.92)$ & \\
\hline$>300.00$ & $1.30(0.91-1.86)$ & & $1.04(0.71-1.54)$ & & $0.86(0.57-1.29)$ & \\
\hline Livestock meat & & 0.082 & & 0.641 & & 0.659 \\
\hline$\leq 100.00$ & 1.00 (reference) & & 1.00 (reference) & & 1.00 (reference) & \\
\hline $100.00-150.00$ & $0.86(0.62-1.18)$ & & $0.86(0.61-1.21)$ & & $0.90(0.63-1.29)$ & \\
\hline$>150.00$ & $0.65(0.44-0.96)^{\star}$ & & $0.89(0.58-1.36)$ & & $0.83(0.53-1.29)$ & \\
\hline Poultry meat & & 0.375 & & 0.642 & & 0.439 \\
\hline$\leq 6.67$ & 1.00 (reference) & & 1.00 (reference) & & 1.00 (reference) & \\
\hline $6.67-16.67$ & $1.37(0.95-1.98)$ & & $1.30(0.87-1.94)$ & & $1.42(0.93-2.17)$ & \\
\hline $16.67-35.71$ & $1.12(0.72-1.73)$ & & $1.22(0.76-1.97)$ & & $1.20(0.73-1.97)$ & \\
\hline$>35.71$ & $1.13(0.71-1.79)$ & & $1.27(0.77-2.10)$ & & $1.31(0.77-2.24)$ & \\
\hline Fish & & $<.0001^{\star}$ & & 0.256 & & 0.355 \\
\hline$\leq 5.00$ & 1.00 (reference) & & 1.00 (reference) & & 1.00 (reference) & \\
\hline $5.00-14.29$ & $0.60(0.41-0.86)^{\star}$ & & $0.80(0.54-1.20)$ & & $0.81(0.53-1.22)$ & \\
\hline $14.29-25.00$ & $0.56(0.37-0.84)$ * & & $0.72(0.46-1.12)$ & & $0.67(0.42-1.08)$ & \\
\hline$>25.00$ & $0.34(0.22-0.54)$ * & & $0.61(0.38-1.00)$ & & $0.67(0.40-1.13)$ & \\
\hline Aquatic product & & $0.008^{*}$ & & 0.822 & & 0.830 \\
\hline$\leq 0.00$ & 1.00 (reference) & & 1.00 (reference) & & 1.00 (reference) & \\
\hline $0.00-0.68$ & $0.70(0.49-0.99)^{\star}$ & & $0.87(0.59-1.27)$ & & $0.83(0.55-1.24)$ & \\
\hline $0.68-2.74$ & $0.62(0.42-0.89) *$ & & $0.86(0.57-1.29)$ & & $0.92(0.60-1.41)$ & \\
\hline$>2.74$ & $0.53(0.35-0.80) \star$ & & $0.96(0.62-1.50)$ & & $0.97(0.60-1.55)$ & \\
\hline \multicolumn{7}{|l|}{ Model 1: crude. } \\
\hline \multicolumn{7}{|c|}{ Model 2: adjusted for age and gender. } \\
\hline \multicolumn{7}{|c|}{$\begin{array}{l}\text { Model 3: further adjusted for education, marital, employment, occupational, alcohol consumption and smoking status, family history of HTN, Famil) } \\
\text { members } \geq 80 \text { years old who are still alive, BMI and pulse. }\end{array}$} \\
\hline
\end{tabular}




\begin{tabular}{|c|c|c|c|c|c|}
\hline Dietary intake & Model 1 & Model 2 & & Model 3 & \\
\hline$\leq 0.00$ & 1.00 (reference) & 1.00 (reference) & & 1.00 (reference) & \\
\hline $0.00-1.94$ & $0.65(0.42-1.00)$ & $0.58(0.36-0.93)^{\star}$ & & $0.62(0.38-1.01)$ & \\
\hline $1.94-3.33$ & $0.53(0.36-0.78)^{\star}$ & $0.55(0.37-0.83)^{\star}$ & & $0.57(0.37-0.88)^{\star}$ & \\
\hline$>3.33$ & $0.95(0.68-1.32)$ & $0.83(0.58-1.19)$ & & $0.83(0.56-1.23)$ & \\
\hline Salt & & & 0.508 & & 0.643 \\
\hline$\leq 3.33$ & 1.00 (reference) & 1.00 (reference) & & 1.00 (reference) & \\
\hline $3.33-4.78$ & $0.75(0.52-1.09)$ & $0.73(0.48-1.10)$ & & $0.77(0.50-1.18)$ & \\
\hline $4.78-5.56$ & $0.64(0.38-1.07)$ & $0.76(0.44-1.33)$ & & $0.79(0.44-1.41)$ & \\
\hline$>5.56$ & $1.04(0.69-1.57)$ & $0.77(0.49-1.21)$ & & $0.76(0.47-1.21)$ & \\
\hline \multicolumn{6}{|l|}{ Model 1: crude. } \\
\hline \multicolumn{6}{|c|}{ Model 2: adjusted for age and gender. } \\
\hline \multicolumn{6}{|c|}{$\begin{array}{l}\text { Model 3: further adjusted for education, marital, employment, occupational, alcohol consumption and smoking status, family history of HTN, Family } \\
\text { members } \geq 80 \text { years old who are still alive, BMI and pulse. }\end{array}$} \\
\hline \multicolumn{6}{|l|}{$\star P<0.05$} \\
\hline
\end{tabular}

\section{Discussion}

This study explored the effects of different kinds of food intake on the prevalence of HTN among Sichuan residents. We found that the intake of eggs was related to the risk of HTN, the daily intake of more than $50 \mathrm{~g}$ egg in the past year might increase the prevalence of HTN. Secondly, we observed that the intake of animal oil was related to the risk of HTN, and the intake of $1.94-3.33 \mathrm{~g}$ of animal oil was beneficial to reduce the risk of HTN. The associations were significant and independent of other confounding factors, mainly including gender, age, BMI and other various dietary factors such as fruit intake, vegetable intake and cereal intake.

Although egg consumption has been found to be a risk factor for HTN, the relationship between egg intake and HTN is complicated and contentious. On the one hand, egg consumption has been inversely associated with the risk of HTN. A study based on a multi-center cohort of black and white Americans revealed that eating more eggs can lower BP and may increase high-density lipoprotein cholesterol (HDL-C), while eating small or moderate amounts of eggs was positively related to total cholesterol and low-density lipoprotein cholesterol (LDL-C) [18]. On the other hand, egg consumption was not related to the risk of HTN. Studies have shown that for patients with coronary heart disease, compared with a typical American breakfast with high calorie or yolk-free egg substitute, daily egg intake had no adverse impact on vascular endothelial function and other heart-related risk measures [19].

In our results, excessive egg consumption was related to a higher incidence of HTN, and the result was contrary to the conclusions of previous publications.

Cholesterol in eggs caused not only an increase in total cholesterol and LDL in serum, but also an increase in HDL [20]. However, excessive intake of eggs may increase the negative effects of total cholesterol and LDL over the positive effects of HDL and phospholipids on HTN, resulting in an increased abnormal rate in BP examination [21]. In addition, unlike western countries, eggs are usually cooked with oil and salt as dishes in China. Adding oil and salt during cooking eggs will increase people's intake of fat and salt. These may be not good for human health.

Our research found that a moderate amount of animal oil intake might be beneficial, and such individuals were less likely to suffer from HTN, while less or more animal oil intake was not significantly associated with the risk of HTN, which was inconsistent with the dietary methods recommended by others [22], but this fact existed objectively. The reasons might come from many sources.

Animal oil for human consumption can be divided into terrestrial animal fats and marine animal fats. Some researchers found, terrestrial animal fats contains a lot of saturated fatty acids (SFA), which may increase the concentration of serum ACE (Angiotensin-converting enzyme), resulting in increased blood pressure [23]. On the other hand, eating terrestrial animal oil with high-fat content for a long time will increase weight and cause obesity, which is also a risk factor for HTN [24]. However, some researches have documented that marine animal fat mainly contains unsaturated fatty acids, which has the effect of regulating blood lipids and blood pressure, and is beneficial to patients with HTN. For patients with HTN, eating high-dose of omega-3 polyunsaturated fatty acid (generally called fish oil) products might help lower BP $[25,26]$. The theoretical study attributes the antihypertensive effect of fish oil to the eicosapentaenoic acid (EPA), which is one of several omega-3 fatty acids commonly used in the human body, and could enhance the beneficial effects of prostacyclin (vasodilator) and reduce the harmful effects of thromboxane (vasoconstrictor) [26]. Therefore, health experts have recommended diets containing less saturated fat and higher polyunsaturated fat [27]. There was no distinction about animal oil in the questionnaire of our study, so the results of this study might be caused by the mixing of oils from terrestrial and marine animals. 
In addition, according to dietary sources, SFA has different carbon lengths and carbon structure [28]. Due to the different types and composition of SFA in each animal oil, its physiological effects are also different [29], the type and source of saturated fat may be as important as the total amount of fat. The national dietary guidelines might treat SFA contained in the diet as a single group of nutrients [30].

In short, the mechanism of dietary influence on HTN is complex and there are serious interactions. To prevent and control HTN, our study suggests that in the daily diet, the crowd should have a reasonable diet, and the food should be diverse. Try to eat low-calorie, low-fat, low-cholesterol, light, easily digestible food, and increase fresh vegetables, fruits, fish, aquatic products, milk and meat consumption, reduce sodium salt, egg and animal oil intake.

There are some limitations to our research. First, because the food frequency questionnaires were limited and inadequate, only the total intake of one type of food can be evaluated instead of specific food, which may lead to a potential impact on the relationship between food consumption and HTN. Second, the dietary intake of the participants was assessed only based on a self-reported questionnaire. Therefore, there might be a reporting bias in the entire cohort. Third, the consumption of the undesirable substances (such as trans fats or acrylamide) produced when cooking have not been fully considered [31] in this study, and they may also lead to an increase in BP. In addition, the study only assessed the dietary intake of participants in the past year, and this dietary habit may change according to the individual's health status. Therefore, assessing a longer-term dietary intake may be more relevant to the current risk of HTN.

\section{Conclusions}

In conclusion, our research shows that excessive consumption of eggs was a risk factor for HTN, while consumption of appropriate amounts of animal oil was a protective factor. However, there is no doubt that more researches are needed to explore the relationships between complex eating habits and HTN risk. Because of the limited number of subjects included in this study, it is necessary to carefully design more high-quality prospective cohort researches to further explore the problems addressed in this study.

\section{Supplementary information}

\section{Abbreviations}

HTN

Hypertension; PPS:Probability proportional to size; SRS:Simple random sampling; SBP:Systolic blood pressure; DBP:Diastolic blood pressure; BMI:Body mass index; SD:Standard deviation; AIC:Akaike's information criteria; OR:Odds ratios; 95\% Cl:95\% Confidence intervals; HDL-C:High-density lipoprotein cholesterol; LDL-C:Low-density lipoprotein cholesterol; SFA:Saturated fatty acids; ACE:Angiotensin-converting enzyme; EPA:Eicosapentaenoic acid.

\section{Declarations}

\section{Ethics approval and consent to participate}

The study has passed the review and approval of the Ethics Committee of Fuwai Hospital (Beijing, China) and obtained the informed consent signed by each participant.

\section{Consent for publication}

Not applicable.

\section{Availability of data and materials}

The datasets analyzed during the current study are available from the corresponding author on reasonable request.

\section{Competing interests}

The authors have stated explicitly that there are no conflict of interest in connection with this article.

\section{Funding}

This study was supported by grants from Wu Jie Ping Medical Foundation Clinical Research Grants Fund (grant no. 320.6750.2020-04-4), Key Research and Development Program of Science and Technology Department of Sichuan Province (grant no. 2019YFS0514), and Research Subject of Health Commission of Sichuan Province (grant no. 19PJ262).

\section{Authors' contributions}

Xingwei Wu: Software, Formal analysis, Writing - Original Draft, Visualization.

Yu Wu: Conceptualization, Investigation, Data curation.

Zhengping Yong: Conceptualization, Investigation, Data curation. 
Zhengyao Hou: Resources, Supervision.

Yueqi Zhang: Resources.

Li Cai, Zhou Liu, Yijia Tang: Investigation.

Rongsheng Tong: Conceptualization, Methodology, Funding acquisition, Writing - Review \& Editing.

Shaoping Wan: Conceptualization, Validation, Project administration, Funding acquisition.

All authors approved the final manuscript for submission.

\section{Acknowledgements}

We are grateful to all participants and staff in this study.

\section{Authors' information (optional)}

${ }^{1}$ Personalized Drug Therapy Key Laboratory of Sichuan Province, School of Medicine, University of Electronic Science and Technology of China, Chengdu 610072, China.

2Department of Pharmacy, Sichuan Academy of Medical Sciences \& Sichuan Provincial People's Hospital, Chengdu 610072, China.

${ }^{3}$ Sichuan Provincial People's Hospital, University of Electronic Science and Technology of China, Chengdu 610072, China.

${ }^{4}$ Sichuan Cancer Hospital \& Institute, Affiliated Hospital of University of Electronic Science and Technology of China, Chengdu, 610041, China.

\section{References}

1. Circulation HJ. Global Disparities of Hypertension Prevalence and Control: A Systematic Analysis of Population-Based Studies From 90 Countries.

2. A comparative risk assessment of burden of. disease and injury attributable to 67 risk factors and risk factor clusters in 21 regions, $1990-2010$ : a systematic analysis for the Global Burden of Disease Study 2010\%J. Lancet. 2012;380:2224.

3. Wang JGJL. A call to action and a lifecourse strategy to address the global burden of raised blood pressure on current and future generations: the Lancet Commission on hypertension.S0140673616311345.

4. WHO. (2013) A Global Brief on Hypertension. https://apps.who.int/iris/bitstream/handle/10665/79059/WHO_DCO_WHD_2013.2_eng.pdf;jsessionid=6C944ADAF819A474804F17FB5326D3EE? sequence=1. Accessed 22 April 2020.

5. Combined impact of risk factors on the subsequent development of hypertension Huang Y, Deng Z, Se Z, Bai Y, Yan C, Zhan Q, Zeng Q, Ouyang P, Dai M, Xu DJJoH. Combined impact of risk factors on the subsequent development of hypertension. 2018.

6. Kwang-Ha YJBO: Association between secondhand smoke exposure and hypertension in never smokers: a cross-sectional survey using data from Korean National Health and Nutritional Examination Survey V. 2010-2012. 8:e021217-

7. Zhang DZJJoHH. Red meat, poultry, and egg consumption with the risk of hypertension: a meta-analysis of prospective cohort studies.

8. Umesawa M, Kitamura A, Kiyama M, Okada T, Shimizu Y, Imano H, Ohira T, Nakamura M, Maruyama K, Iso HJHR. Association between dietary behavior and risk of hypertension among Japanese male workers. 36:374-380.

9. Appel LJ, Moore TJ, Obarzanek E, Vollmer WM, Svetkey LP, Sacks FM, Bray GA, Vogt TM, Cutler JA, Windhauser MM, et al. A clinical trial of the effects of dietary patterns on blood pressure. DASH Collaborative Research Group. N Engl J Med. 1997;336:1117-24.

10. Saneei P, Salehi-Abargouei A, Esmaillzadeh A, Azadbakht LJNM, Nmcd CD. Influence of Dietary Approaches to Stop Hypertension (DASH) diet on blood pressure: A systematic review and meta-analysis on randomized controlled trials. 24:1253-1261.

11. Soner D. Rational approaches to the treatment of hypertension: diet.

12. H GA. F, P M, A D, E Y, Hypertension AFJHrojotJSo: Adherence to the Mediterranean diet is associated with reduced risk of incident chronic kidney diseases among Tehranian adults. 2017, 40:96-102.

13. Worldwide trends in blood pressure. from 1975 to 2015 : a pooled analysis of 1479 population-based measurement studies with $19 \cdot 1$ million participants. Lancet. 2017;389:37-55.

14. N GYHFWH. Z, D D, G L, hypertension LHJAjo: A Dietary Pattern of Higher Fish, Egg, Milk, Nut, Vegetable and Fruit, and Lower Salt Intake Correlates With the Prevalence and Control of Hypertension. 2018, 31:679-686.

15. Wang Z, Chen Z, Zhang L, Wang X, Hao G, Zhang Z, Shao L, Tian Y, Dong Y. Zheng CJä,: Status of Hypertension in China: Results from the China Hypertension Survey, 2012-2015.CIRCULATIONAHA.117.032380.

Page $12 / 14$ 
16. China Hypertension Control Guidelines Revision Committee. Alliance H, Chinese Medical Association Cardiology Branch, et al. Chinese Hypertension Prevention and treatment guidelines (2018 revision). Chinese Journal of Cardiovascular Medicine. 2019;24(1):24-56.

17. Chen Z, Wang X, Wang Z, Zhang L, Hao G, Dong Y, Zhu M. Gao RJä,: Assessing the validity of oscillometric device for blood pressure measurement in a large population-based epidemiological study. 032:269-270.

18. Meyer KA. Abstract P286: Egg Consumption is Associated With Cardiovascular Disease Risk Factors in the Coronary Artery Risk Development in Young Adults Study. 2017, 135:AP286-AP286.

19. Katz DL, Gnanaraj J, Treu JA, Ma Y, Kavak Y, Njike VY. Effects of egg ingestion on endothelial function in adults with coronary artery disease: a randomized, controlled, crossover trial. Am Heart J. 2015;169:162-9.

20. Samantha B, Gowri R, Rohini V, Jacques PF, Johnson EJJAJoCN. Dietary cholesterol and cardiovascular disease: a systematic review and metaanalysis. 2015:2.

21. G HHTZJZZZXNGZ Z, reports LAJS: Associations of physical activity and egg intake with hypertension among Chinese middle-aged and older population. 2019, 9:7722.

22. Eckel RH, Jakicic JM, Ard JD, de Jesus JM, Houston Miller N, Hubbard VS, Lee IM, Lichtenstein AH, Loria CM, Millen BE, et al. 2013 AHA/ACC guideline on lifestyle management to reduce cardiovascular risk: a report of the American College of Cardiology/American Heart Association Task Force on Practice Guidelines. J Am Coll Cardiol. 2014;63:2960-84.

23. Schuler R, Osterhoff MA, Frahnow T, Seltmann AC, Busjahn A, Kabisch S, Xu L, Mosig AS, Spranger J, Mohlig M, et al: High-Saturated-Fat Diet Increases Circulating Angiotensin-Converting Enzyme, Which Is Enhanced by the rs4343 Polymorphism Defining Persons at Risk of NutrientDependent Increases of Blood Pressure. J Am Heart Assoc 2017, 6.

24. Hooper L, Abdelhamid A, Bunn D, Brown T, Summerbell CD, Skeaff CM. Effects of total fat intake on body weight. Cochrane Database Syst Rev 2015:Cd011834.

25. Appel LJ, Miller ER 3rd, Seidler AJ, Whelton PK. Does supplementation of diet with 'fish oil' reduce blood pressure? A meta-analysis of controlled clinical trials. Arch Intern Med. 1993;153:1429-38.

26. Morris MC, Sacks F, Rosner B. Does fish oil lower blood pressure? A meta-analysis of controlled trials. Circulation. 1993;88:523-33.

27. Healthy. Diet-World Health Organization.

28. Bainbridge ML, Cersosimo LM, Wright AD, Kraft JJPO. Content and Composition of Branched-Chain Fatty Acids in Bovine Milk Are Affected by Lactation Stage and Breed of Dairy Cow. 2016, 11:e0150386.

29. Miguel AJJCRiFS. Nutrition: The Food Matrix: Implications in Processing, Nutrition and Health.1-43.

30. Unger AL, Torres-Gonzalez M, Kraft J. Dairy Fat Consumption and the Risk of Metabolic Syndrome: An Examination of the Saturated Fatty Acids in Dairy. Nutrients 2019, 11.

31. Liska DAJ, Cook CM, Wang DD, Szpylka JJFS. Nutrition: Maillard reaction products and potatoes: have the benefits been clearly assessed?, 4:234249.

32. Publisher's Note.

33. Springer. Nature remains neutral with regard to jurisdictional claims in published maps and institutional affiliations.

\section{Figures}




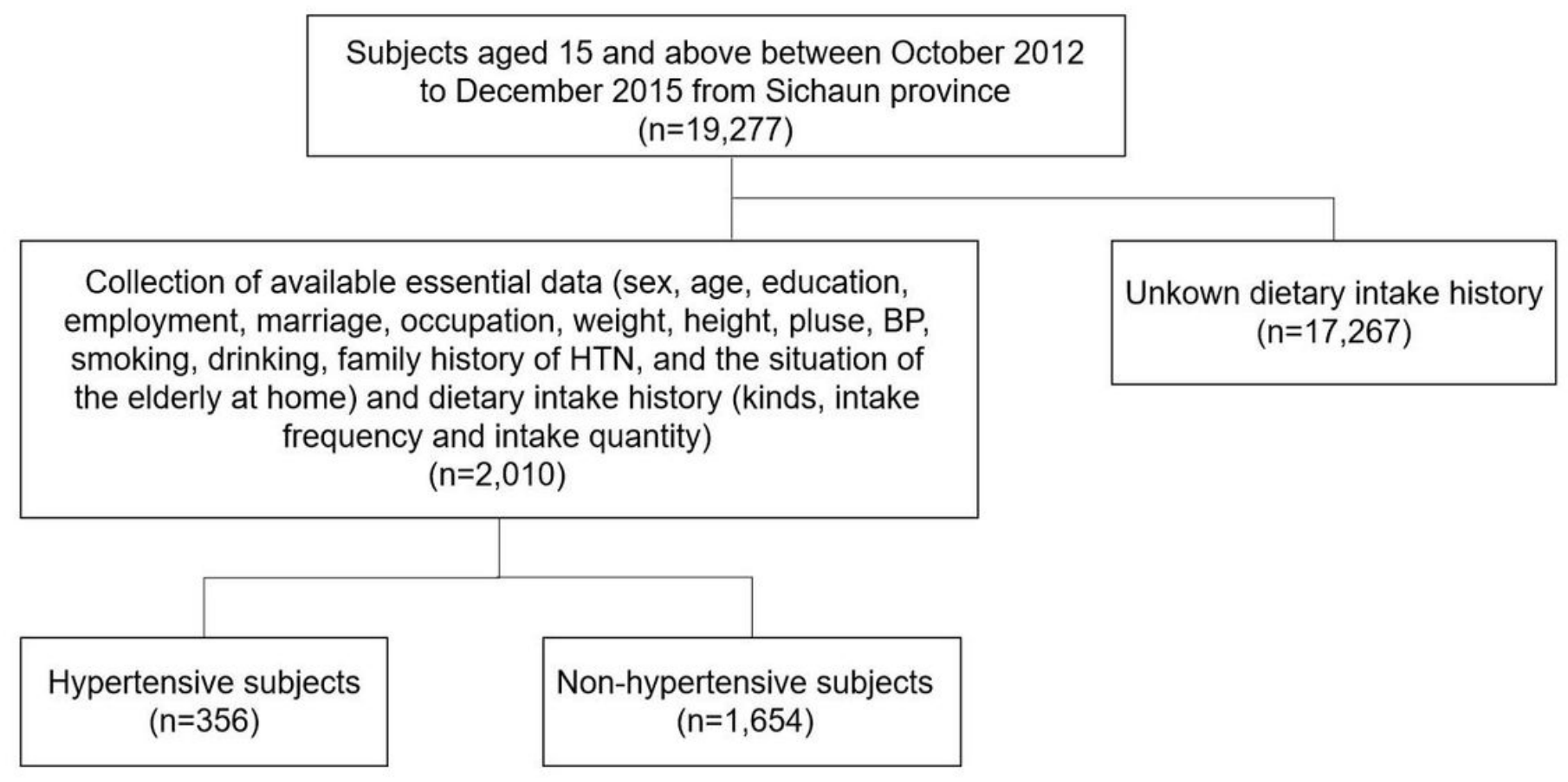

Figure 1

Flow diagram of study population. Data of 19,277 subjects in Sichuan were obtained, and 17,267 subjects with incomplete dietary intake history were excluded. Finally, A total of 2,010 subjects were included in this study, including 356 subjects with hypertension.

\section{Supplementary Files}

This is a list of supplementary files associated with this preprint. Click to download.

- renamed58290.docx 